\title{
Methoden
}

Sönke Rhein*, Tilman Utz und Knut Graichen

\section{Dynamische Optimierung von Multiphysik-Problemen am Beispiel induktiver Heizvorgänge}

\author{
Dynamic optimization of multiphysics problems applied to induction heating processes
}

DOI 10.1515/auto-2015-0029

Eingang 13. März 2015; angenommen 27. Mai 2015

Zusammenfassung: Der Beitrag stellt einen Ansatz zur dynamischen Optimierung von Multiphysik-Problemen am Beispiel induktiver Heizvorgänge vor. Hierfür werden im Rahmen eines first-optimize-then-discretize-Ansatzes zunächst die Optimalitätsbedingungen in Form von partiellen Differentialgleichungen aufgestellt. Dies ermöglicht eine elegante numerische Lösung des Problems durch den Einsatz eines Gradientenverfahrens in Verbindung mit FEM-Software (FEM - Finite-Elemente-Methode). Neben der Auslagerung des numerischen Aufwands liegen die Vorteile des Ansatzes insbesondere darin, dass auch Probleme auf komplexen Geometrien relativ einfach behandelt werden können. Die geeignete Formulierung und numerische Lösung des Optimierungsproblems wird anhand von Simulationsergebnissen für die induktive Aufheizung und Oberflächenhärtung eines Zahnrads präsentiert.

Schlüsselwörter: Multiphysik-Probleme, dynamische Optimierung, formale Lagrange-Technik, induktives Heizen.

Abstract: This contribution presents a dynamic optimization scheme for multiphysics problems applied to an inductive heating application. A first optimize then discretize approach is used in order to derive the optimality conditions of the original problem in terms of partial differential equations. This allows for an elegant numerical solution by using a gradient method in combination with FEM software (FEM - finite element method). This approach has the advantage of outsourcing the numerical integrations of the optimization algorithm to FEM software and to enable

\footnotetext{
*Korrespondenzautor: Sönke Rhein, Universität Ulm, Ulm, E-Mail: soenke.rhein@uni-ulm.de

Tilman Utz, Knut Graichen: Universität Ulm, Ulm
}

the handling of complex geometries with relative ease. The approach is presented in simulation studies for the inductive heating and surface hardening of a gear wheel.

Keywords: Multiphysics problems, dynamic optimization, formal Lagrangian technique, induction heating.

MSC: 35Q93, 49M05, 35Q61, 78M10

Gewidmet Herrn Univ.-Prof. Dr. techn. Kurt Schlacher zu seinem 60. Geburtstag

\section{Einleitung}

Eine hinreichend genaue mathematische Beschreibung physikalischer Vorgänge erfordert vielmals die simultane Berücksichtigung einer zeitlichen und örtlichen Dynamik. Häufig umfassen dabei solche sogenannte verteiltparametrischen Systeme unterschiedliche und in einer wechselseitigen Beziehung stehende physikalische Effekte, die mathematisch durch verkoppelte partielle Differentialgleichungen (pDGln) beschrieben werden können. In diesem Zusammenhang spricht man auch von Multiphysik-Problemen [14]. Typische Beispiele sind die Tumorbehandlung durch Bestrahlung [19], die Untersuchung von Schallausbreitung [16], die Prozessführung chemischer Reaktoren [25] oder induktive Heizvorgänge $[21,24,27]$.

Außer für sehr realitätsnahe Simulationen, zum Beispiel zur Erlangung eines tieferen Systemverständnisses [3], besteht zunehmend auch ein regelungstechnisches Interesse an Multiphysik-Problemen [5, 13, 23]. Hervorgerufen durch die verteilt-parametrische Systemdynamik sind die damit verbundenen Herausforderungen zu einem Großteil auch in numerischen Aspekten zu sehen. Dies trifft insbesondere dann zu, wenn komplexe Ortsgeometrien berücksichtigt werden müssen. 
Speziell bei Multiphysik-Problemen kommt je nach zugrundeliegenden physikalischen Effekten die Schwierigkeit hinzu, die Systemdynamik unter Beachtung teilweise stark unterschiedlicher Längen- und Zeitskalen numerisch lösen zu müssen [14]. Weiterhin führt die Betrachtung unterschiedlicher physikalischer Domänen häufig zu einer verkoppelten Systemdynamik.

Als eine besonders gut geeignete Methode für den Reglerentwurf von verteilt-parametrischen Systemen hat sich in den letzten Jahrzehnten die dynamische Optimierung etabliert $[6,13,15]$. Hierbei wird im Rahmen eines Optimalsteuerungsproblems das Regelungsziel durch ein Gütefunktional abgebildet und die zugrundeliegende Systemdynamik in Form von dynamischen Gleichungsbeschränkungen berücksichtigt [23]. Infolgedessen kann der Reglerentwurf auf die numerische Lösung des Optimalsteuerungsproblems verlagert werden, wobei hierfür die beiden Ansätze first optimize then discretize (FOTD) und first discretize then optimize (FDTO) zur Verfügung stehen [13].

Bei einem FDTO-Ansatz wird zunächst die Systemdynamik diskretisiert, um anschließend die approximierte Problemstellung mit bewährten Methoden und Algorithmen der klassischen Optimierung handhaben zu können [4]. Im Falle komplexerer Ortsgeometrien wächst jedoch bei diesem Ansatz der nötige Diskretisierungsaufwand sehr schnell an und erfordert einen stark problemspezifischen Lösungsansatz.

Die Idee eines FOTD-Ansatzes besteht darin, die Optimalitätsbedingungen direkt in einer verteiltparametrischen Systemformulierung aufzustellen. Dies führt dazu, dass das sogenannte adjungierte System ebenfalls in Form einer pDGl bzw. eines pDGl-Systems vorliegt [13]. Anschließend werden die Optimalitätsbedingungen für die numerische Lösung des Optimalsteuerungsproblems diskretisiert.

In diesem Beitrag wird am Beispiel induktiver Heizvorgänge gezeigt, wie durch die Anwendung eines FOTD-Ansatzes der mit der dynamischen Optimierung von Multiphysik-Problemen einhergehende numerische Aufwand maßgeblich auf FEM-Software (FEM - FiniteElemente-Methode) ausgelagert werden kann. Hierfür wird durch die geeignete Formulierung eines dynamischen Optimierungsproblems dafür gesorgt, dass eine sequentielle Lösung der Systemdynamik und der adjungierten Dynamik im Rahmen eines Gradientenverfahrens durch die FEM-Software ermöglicht wird [9]. Die benötigten Optimalitätsbedingungen werden dabei mit Hilfe der formalen Lagrange-Technik hergeleitet [23].

Der in diesem Beitrag verfolgte Ansatz führt dazu, dass sowohl die Systemdynamik als auch die adjungierte Dynamik in Form eines pDGl-Systems vorliegt und je- weils mit Hilfe von FEM-Software numerisch gelöst werden kann. Für das betrachtete Anwendungsbeispiel des induktiven Heizens können somit auch komplexere Ortsgeometrien wie Zahnräder ohne größeren Aufwand berücksichtigt werden. Weiterhin wird gezeigt, dass je nach Formulierung des Gütefunktionals unterschiedliche Problemstellungen wie das Aufheizen auf örtlich konstante Temperaturprofile oder Oberflächenhärtungsvorgänge realisiert werden können.

Der Beitrag ist wie folgt gegliedert. In Abschnitt 2 wird das Multiphysik-Problem des induktiven Heizens in Form eines verkoppelten pDGl-Systems angegeben. Die Formulierung eines geeigneten dynamischen Optimierungsproblems mit zwei unterschiedlichen Gütefunktionalen zur Realisierung von Aufheiz- und Oberflächenhärtungsvorgängen wird in Abschnitt 3 vorgestellt. Weiterhin werden unter Verwendung der formalen Lagrange-Technik die Optimalitätsbedingungen des Optimierungsproblems gemäß eines FOTD-Ansatzes hergeleitet, um dieses anschließend in Abschnitt 4 im Rahmen eines Gradientenverfahrens mit Hilfe von FEM-Software numerisch lösen zu können. In Abschnitt 5 werden Simulationsergebnisse für induktive Aufheiz- und Oberflächenhärtungsvorgänge am Beispiel eines Zahnrads präsentiert. Der Beitrag endet mit Abschnitt 6 in Form einer kurzen Zusammenfassung und einem Ausblick.

\section{Systembeschreibung}

In diesem Abschnitt wird das im weiteren Verlauf betrachtete Multiphysik-Problem des induktiven Heizens vorgestellt. Hierfür wird neben einer kurzen Beschreibung der Regelungsziele die zugrundeliegende Systemdynamik in Form eines pDGl-Systems angegeben.

\subsection{Induktive Heizvorgänge}

Vorrichtungen zur induktiven Erwärmung metallischer Werkstücke bestehen im Wesentlichen aus einer Spule und dem zu erhitzenden Werkstück selbst und sind damit relativ einfach aufgebaut. Andererseits basieren induktive Heizvorgänge auf stark verkoppelten elektromagnetischen, thermodynamischen und metallurgischen Vorgängen. Beim Thixoforming zum Beispiel wird ein Werkstück induktiv auf ein örtlich konstantes Temperaturprofil aufgeheizt und dadurch in einen teilflüssigen Aggregatzustand gebracht, wodurch ein anschließender Formgebungsprozess erleichtert wird [7]. Bei Härtungsvorgängen hingegen wird lediglich die Werkstücksoberfläche partiell 


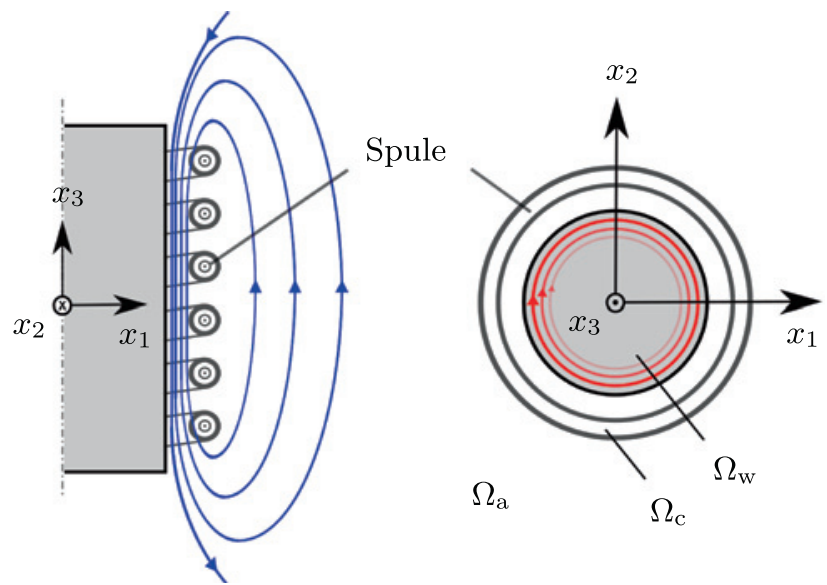

Abbildung 1: Schematischer Aufbau eines induktiven Heizvorgangs mit zylindrischem Werkstück. Längsschnitt mit angedeutetem Magnetfeld (links) und Draufsicht mit induzierten Strömen (rechts).

aufgeheizt und wieder rasch abgekühlt, um dort eine Martensitbildung zu ermöglichen [21].

Da aus regelungstechnischer Sicht gesehen die erwünschten metallurgischen Vorgänge und Eigenschaften durch eine geeignete Einstellung zeit- und ortsabhängiger Profile der Werkstücktemperatur herbeigeführt werden können, reduziert sich das zu betrachtende MultiphysikProblem auf eine elektromagnetische sowie eine thermische Komponente [21]. Dabei wird mit Hilfe der Spule ein magnetisches Wechselfeld aufgebaut, das in dem im Magnetfeld platzierten Werkstück Ströme induziert. Infolge der damit einhergehenden Joule'schen Verluste wird das Werkstück erhitzt. Eine hierfür typische Vorrichtung zur induktiven Erwärmung eines zylindrischen Werkstücks ist in Abbildung 1 zu sehen.

Eine besondere Herausforderung ergibt sich bei komplexeren Werkstückgeometrien, da diese zu einer örtlich stark unterschiedlichen Verteilung der induzierten Ströme führen. Um der damit einhergehenden ungleichen Erwärmung des Werkstücks durch eine geeignete Ansteuerung der Spule entgegentreten zu können, wird im weiteren Verlauf dieses Beitrags ein dynamisches Optimierungsproblem betrachtet. Die Modellierung der zu beachtenden Systemdynamik des induktiven Heizvorgangs in Form eines elektromagnetischen sowie eines thermischen Teilsystems ist Bestandteil der folgenden beiden Abschnitte.

\subsection{Modellierung der Systemdynamik}

Im Folgenden wird in Anlehnung an Abbildung 1 die Geometrie des Werkstücks und der Spule mit Hilfe kartesischer Koordinaten $x:=\left(x_{1}, x_{2}, x_{3}\right)^{\top}$ durch die Gebiete $\Omega_{\mathrm{w}}$ und $\Omega_{\mathrm{c}}$ mit $\Omega_{\mathrm{w}} \cap \Omega_{\mathrm{c}}=\emptyset$ beschrieben. Der Raum zwischen der Spule und dem Werkstück sowie die Umgebung wird zum Gebiet $\Omega_{\mathrm{a}}$ zusammengefasst. Während für die elektromagnetische Problemstellung das gesamte Gebiet $\Omega=$ $\Omega_{\mathrm{w}} \cup \Omega_{\mathrm{c}} \cup \Omega_{\mathrm{a}}$ mit entsprechendem Rand $\partial \Omega$ betrachtet werden muss, kann die thermische Problemstellung auf das Gebiet $\Omega_{\mathrm{w}}$ mit dem Rand $\partial \Omega_{\mathrm{w}}$ beschränkt werden.

\subsubsection{Elektromagnetisches Teilsystem}

Für die mathematische Modellierung des elektromagnetischen Teilsystems wird im Folgenden der lineare Zusammenhang

$$
\mathcal{B}=\mu \mathcal{H} \quad \text { in } \Omega
$$

zwischen der magnetischen Flussdichte $\mathcal{B}:=\mathcal{B}(x, t)$ und der magnetischen Feldstärke $\mathcal{H}:=\mathcal{H}(x, t)$ mit der Proportionalitätskonstante $\mu:=\mu_{0} \mu_{\mathrm{r}}(x)$ angenommen [21]. Zudem wird von einer harmonischen Anregung des Magnetfelds durch den Spulenstrom

$$
\mathcal{J}_{c}:=\mathcal{J}_{c}(x, t)=\hat{\mathcal{J}}_{\mathrm{c}} \cos (\omega t) \quad \text { in } \Omega_{\mathrm{c}}
$$

mit der Amplitude $\hat{\mathcal{J}}_{c}:=\hat{\mathcal{J}}_{c}(x)$ bei einer Kreisfrequenz $\omega$ von unter $10 \mathrm{MHz}$ ausgegangen, wodurch Verschiebungsströme vernachlässigt werden können [21].

Die elektromagnetische Problemstellung kann dann unter Annahme geeigneter Randbedingungen für die elektrischen und magnetischen Feldgrößen durch die quasistationären harmonischen Maxwell-Gleichungen

$$
\begin{array}{ll}
\operatorname{curl} \mathcal{H}=\sigma \mathcal{E}+\mathcal{J}_{\mathrm{c}} & \text { in } \Omega \\
\operatorname{curl} \mathcal{E}=-\partial_{t} \mathcal{B} & \text { in } \Omega
\end{array}
$$

mit der elektrischen Feldstärke $\mathcal{E}:=\mathcal{E}(x, t)$ und der elektrischen Leitfähigkeit $\sigma:=\sigma(x)$ beschrieben werden [1].

Eine numerisch günstigere Formulierung der Maxwell-Gleichungen (3) kann mit Hilfe des magnetischen Vektorpotentials $\mathcal{A}:=\mathcal{A}(x, t)$ erzielt werden $[12,17]$. Dieses ist durch

$$
\mathcal{B}=\operatorname{curl} \mathcal{A} \quad \text { in } \Omega
$$

definiert und garantiert gleichzeitig die Quellenfreiheit des Magnetfelds. Wird weiterhin der komplexe Phasor $A:=A(x)$ mit $\mathcal{A}(x, t)=\operatorname{Re}\left\{A(x) e^{j \omega t}\right\}$ und der imaginären Einheit $j$ verwendet, so kann anstelle von (3) die sowohl entkoppelte als auch nicht mit zeitlichen dynamischen Vorgängen behaftete Problemstellung

$$
\begin{array}{ll}
\operatorname{curl}\left(\mu^{-1} \operatorname{curl} A\right)+j \omega \sigma A=J_{\mathrm{c}}(u(t)) & \text { in } Q \\
A \times \vec{n}=0 & \text { auf } \Sigma
\end{array}
$$


auf den Orts-Zeit-Zylindern $Q:=\Omega \times\left(0, t_{\mathrm{f}}\right)$ und $\Sigma:=\partial \Omega \times$ $\left(0, t_{\mathrm{f}}\right)$ mit der Endzeit $t_{\mathrm{f}}>0$ betrachtet werden.

Die hinzugekommene Randbedingung (5b) des elektromagnetischen Problems mit dem nach außen gerichteten Normalenvektor $\vec{n}$ beruht auf der Annahme einer verschwindenden Tangentialkomponente des magnetischen Vektorpotentials $A$ auf dem Rand $\partial \Omega$. Im Rahmen der numerischen Lösung wird dies durch eine hinreichend große Entfernung zwischen dem Gebiet $\Omega_{\mathrm{c}}$ und dem Rand $\partial \Omega$ berücksichtigt.

Bei der Formulierung des elektromagnetischen Problems (5) wird mit den Orts-Zeit-Zylindern $Q$ und $\Sigma$ eine zeitlich abhängige Amplitude der Spulenstromdichte

$$
J_{\mathrm{c}}(u(t))= \begin{cases}\frac{N_{\mathrm{c}}}{A_{\mathrm{c}}} \vec{e}_{\mathrm{c}} u(t) & \text { falls } x \in \Omega_{\mathrm{c}} \\ (0,0,0)^{\top} & \text { sonst }\end{cases}
$$

angenommen, wodurch im weiteren Verlauf dieses Beitrags eine Steuerung der induktiven Heizvorgänge mit der Stellgröße $u:=u(t)$ ermöglicht wird. Die Richtung des Stromflusses in der zylindrischen Spule mit der Windungszahl $N_{\mathrm{c}}$ und dem Gesamtleiterquerschnitt $A_{\mathrm{c}}$ ist durch den Vektor

$$
\vec{e}_{\mathrm{c}}=\left(\frac{-x_{2}}{\sqrt{x_{1}^{2}+x_{2}^{2}}}, \frac{x_{1}}{\sqrt{x_{1}^{2}+x_{2}^{2}}}, 0\right)^{\top}
$$

beschrieben. Die Frequenz des eingeprägten Stroms wird als konstant angenommen, aber dem jeweiligen Problem angepasst.

\subsubsection{Thermisches Teilsystem}

Die im Werkstück induzierten Ströme $J_{\mathrm{i}}=j \omega \sigma A$ führen zu Joule'schen Verlusten $P_{\mathrm{i}}=\frac{\sigma \omega^{2}}{2}|A|^{2}$, wobei $|A|^{2}=$ $\operatorname{Re}\{A\}^{2}+\operatorname{Im}\{A\}^{2}$ gilt. Somit kann die Temperaturverteilung innerhalb des Werkstücks mit Hilfe der nichtlinearen Wärmeleitungsgleichung

$$
\begin{array}{lr}
\rho C_{\mathrm{p}} \partial_{t} T-\nabla \cdot(k \nabla T)=\frac{\sigma \omega^{2}}{2}|A|^{2} & \text { in } \mathrm{Q}_{\mathrm{w}} \\
\vec{n} \cdot(k \nabla T)=\alpha\left(T_{\mathrm{a}}-T\right)+\epsilon \sigma_{\mathrm{SB}}\left(T_{\mathrm{a}}^{4}-T^{4}\right) & \text { auf } \Sigma_{\mathrm{w}} \\
T(\cdot, 0)=T_{0} & \text { in } \Omega_{\mathrm{w}}
\end{array}
$$

mit der Temperatur $T:=T(x, t)$ auf den Orts-ZeitZylindern $Q_{\mathrm{w}}:=\Omega_{\mathrm{w}} \times\left(0, t_{\mathrm{f}}\right)$ und $\Sigma_{\mathrm{w}}:=\partial \Omega_{\mathrm{w}} \times\left(0, t_{\mathrm{f}}\right)$ modelliert werden.

Mit der Randbedingung (8b) lässt sich sowohl eine konvektive Wärmeübertragung als auch eine Abstrahlung in die Umgebung modellieren. Hierbei wird jeweils eine konstante Umgebungstemperatur $T_{\mathrm{a}}$ angenommen, wo- bei die konvektive Wärmeübertragung mit dem Wärmeübergangskoeffizient $\alpha$ und die strahlungsbehaftete Wärmeübertragung mit Hilfe des Emissionsparameters $\epsilon$ und der Stefan-Boltzmann-Konstante $\sigma_{\mathrm{SB}}$ beschrieben ist. Die Anfangsbedingung (8c) der Wärmeleitungsgleichung ist durch das Temperaturprofil $T_{0}:=T(x, 0)$ bestimmt.

In vielen praktischen Anwendungen ist die Annahme, dass die Dichte $\rho:=\rho(x)$, die spezifische Wärmekapazität $C_{\mathrm{p}}:=C_{\mathrm{p}}(x)$, die Wärmeleitfähigkeit $k:=k(x)$ sowie die elektrische Leitfähigkeit $\sigma:=\sigma(x)$ unabhängig von der Temperatur $T$ ist, eine zulässige Annahme [21]. Diese Annahme wird hier übernommen, um die prinzipielle Vorgehensweise zur Herleitung der Optimalitätsbedingungen im folgenden Abschnitt in einfacherer Weise darstellen zu können. Insbesondere wenn das behandelte Werkstück kristalline Veränderungen durchläuft, wie es zum Beispiel bei der Oberflächenhärtung zu erwarten ist, muss diese Annahme sowie die daraus resultierende Anpassungen der Optimalitätsbedingungen überprüft werden.

\section{Dynamische Optimierung}

Im Folgenden werden zwei verschiedene Varianten eines dynamischen Optimierungsproblems zur Realisierung von Aufheiz- und Oberflächenhärtungsvorgängen vorgestellt. Die Herleitung der Optimalitätsbedingungen gemäß eines FOTD-Ansatzes unter Verwendung der formalen LagrangeTechnik ermöglicht die numerische Lösung der betrachteten Problemstellungen im nächsten Abschnitt.

\subsection{Problemformulierung}

Der Grundgedanke der dynamischen Optimierung besteht in der Formulierung und numerischen Lösung eines Optimierungsproblems, wobei die Regelungsziele durch ein Gütefunktional abgebildet werden können [13]. Für die in diesem Beitrag betrachteten induktiven Aufheiz- und Oberflächenhärtungsvorgänge sollen dabei sowohl die Eingangsbeschränkungen

$$
u \in \mathcal{U}_{\mathrm{ad}}:=\left\{u \in \mathbb{R} \mid u^{-} \leq u \leq u^{+}\right\}
$$

mit den Schranken $0 \leq{u^{-}}^{-}<u^{+}$als auch die Zustandsbeschränkung

$$
T \in \mathcal{T}_{\text {ad }}:=\left\{T \in \mathbb{R} \mid T \leq T^{+}\right\}
$$

mit der maximal zulässigen Werkstücktemperatur $T^{+}$zur Verhinderung einer Schädigung des Werkstücks durch Überhitzung eingehalten werden [21]. 
Während die formulierten Eingangsbeschränkungen im Rahmen der numerischen Lösung des Optimierungsproblems in der Regel ohne größeren Aufwand berücksichtigt werden können, würde die direkte Berücksichtigung der Zustandsbeschränkung (10) mit einem relativ hohen Aufwand einhergehen. Dies trifft sowohl auf die Herleitung der Optimalitätsbedingungen als auch auf den nötigen Aufwand für deren numerische Lösung zu [18].

Eine geeignete Wahl des Gütefunktionals für die in diesem Beitrag betrachteten induktiven Heizvorgänge ist durch

$$
\begin{aligned}
& J(T, u)=\underbrace{\int_{\Omega_{\mathrm{w}}} \frac{q_{1}}{2} \zeta(x)\left(T\left(\cdot, t_{\mathrm{f}}\right)-T_{\mathrm{d}}\right)^{2} \mathrm{~d} x+}_{=: V\left(T\left(\cdot, t_{\mathrm{f}}\right)\right)} \\
& \int_{0}^{\int_{\mathrm{f}}} \underbrace{\int_{\Omega_{\mathrm{w}}} \frac{q_{2}}{2} \zeta(x)\left(T-T_{\mathrm{d}}\right)^{2}+\frac{q_{3}}{2} \max \left(0, T-T^{+}\right)^{2} \mathrm{~d} x+\frac{q_{4}}{2} u^{2} \mathrm{~d} t}_{=: l(T, u)}
\end{aligned}
$$

mit dem Mayer-Term $V\left(T\left(\cdot, t_{\mathrm{f}}\right)\right)$ und dem LagrangeAnteil $l(T, u)$ gegeben. Durch die spezielle Wahl der Funktion $\zeta(x)$ können dabei sowohl die Anforderungen für reine Aufheizvorgänge auf örtlich konstante Temperaturprofile als auch für Oberflächenhärtungsvorgänge mit einer lediglich partiellen Erwärmung der Randschicht formuliert werden.

Für die Aufheizvorgänge wird die quadratische Bestrafung der Abweichung der Temperatur $T$ zur Solltemperatur $T_{\mathrm{d}}$ mit Hilfe des Mayer-Terms $V\left(T\left(\cdot, t_{\mathrm{f}}\right)\right)$ und dem Lagrange-Anteil $l(T, u)$ auf dem gesamten Gebiet $\Omega_{\mathrm{w}}$ berücksichtigt, d.h., es gilt

$$
\zeta(x)=1 \quad \forall x \in \Omega_{\mathrm{w}} .
$$

Eine geeignete Wahl für die Oberflächenhärtung ist hingegen durch

$$
\zeta(x)= \begin{cases}1 & \text { falls } x \in \tilde{\Omega}_{\mathrm{w}} \\ 0 & \text { sonst }\end{cases}
$$

mit dem Bereich der zu härtenden Randschicht

$$
\tilde{\Omega}_{\mathrm{w}}:=\left\{x \in \Omega_{\mathrm{w}} \mid\|x-y\|_{2}^{2} \leq \delta_{\mathrm{p}}^{2} \quad \forall y \in \partial \Omega_{\mathrm{w}}\right\}
$$

gegeben. Die Tiefe der zu härtenden Randschicht wird über $\delta_{\mathrm{p}}>0$ eingestellt.

Neben der Abbildung der Regelungsziele durch die spezielle Wahl der Funktion $\zeta(x)$ beinhaltet das Gütefunktional (11) eine äußere Straffunktion zur indirekten Berücksichtigung der Zustandsbeschränkung (10), wodurch
Temperaturen oberhalb von $T^{+}$bestraft werden. Weiterhin ist im Gütefunktional eine Gewichtung der Stellgröße $u$ enthalten, um den Energieeintrag zu berücksichtigen. Die einzelnen Anteile werden mit den Faktoren $q_{\mathrm{i}} \geq 0, i \in$ $\{1, \ldots, 4\}$ gewichtet.

Für die dynamische Optimierung der induktiven Aufheiz- und Oberflächenhärtungsvorgänge kann somit das Optimierungsproblem

$$
\begin{array}{cc}
\min _{u \in \mathcal{U}_{\mathrm{ad}}} J(T, u)=V\left(T\left(\cdot, t_{\mathrm{f}}\right)\right)+\int_{0}^{t_{\mathrm{f}}} l(T, u) \mathrm{d} t & \\
\text { u.B.v.: } \rho C_{\mathrm{p}} \partial_{t} T-\nabla \cdot(k \nabla T)=\frac{\sigma \omega^{2}}{2}|A|^{2} & \text { in } \mathrm{Q}_{\mathrm{w}} \\
\vec{n} \cdot(k \nabla T)=\alpha\left(T_{\mathrm{a}}-T\right) & \\
\quad+\epsilon \sigma_{\mathrm{SB}}\left(T_{\mathrm{a}}^{4}-T^{4}\right) & \text { auf } \Sigma_{\mathrm{w}} \\
T(\cdot, 0)=T_{0} & \text { in } \Omega_{\mathrm{w}} \\
\operatorname{curl}\left(\mu^{-1} \operatorname{curl} A\right)+j \omega \sigma A=J_{\mathrm{c}}(u) & \text { in } Q \\
A \times \vec{n}=0 & \text { auf } \Sigma
\end{array}
$$

angegeben werden. Bei der numerischen Lösung der Systemdynamik (15b)-(15f) ist die instantane Ausbreitung des elektromagnetischen Feldes im Gegensatz zu der vergleichsweise langsam ablaufenden Wärmeleitung $\mathrm{zu}$ beachten. Infolgedessen beinhaltet das zu betrachtende Optimierungsproblem ein partielles differentialalgebraisches Gleichungssystem, was bei der numerischen Lösung durch die Wahl geeigneter Algorithmen berücksichtigt werden muss.

Eine Herausforderung im Zusammenhang mit der numerischen Lösung des Optimierungsproblems (15) sind auch die stark unterschiedlichen Längenskalen des elektromagnetischen und thermischen Problems. Vor allem der zu beachtende Skin-Effekt führt dazu, dass sich mit höheren Kreisfrequenzen $\omega$ die induzierten Ströme überwiegend in den Randbereichen des zu erhitzenden Werkstücks konzentrieren und somit durch immer feinere Ortsdiskretisierungen aufgelöst werden müssen [1]. Im Falle komplexerer Ortsgeometrien kommt erschwerend hin$\mathrm{zu}$, dass sogenannte End- und Edge-Effekte zu einer stark unterschiedlichen Verteilung der induzierten Ströme entlang der Randbereiche führen. Für eine ausführliche Diskussion hinsichtlich der zu erwartenden Stromverteilung, vor allem im Bezug auf komplexere Ortsgeometrien, wird auf [21] verwiesen.

\subsection{Optimalitätsbedingungen}

Zunächst werden die schwachen Formulierungen der Systemdynamik (15b)-(15d) bzw. (15e)-(15f) bestimmt. Die- 
se werden dann aufgegriffen, um mit Hilfe der formalen Lagrange-Technik die zum Optimierungsproblem (15) zugehörigen Optimalitätsbedingungen in Form von zwei gekoppelten adjungierten Systemen sowie eines Gradienten herzuleiten.

\subsubsection{Schwache Formulierung der Systemdynamik}

Für die Bestimmung der schwachen Formulierung des thermischen Teilsystems (15b)-(15d) wird Gleichung (15b) mit der Testfunktion $\Phi:=\Phi(x, t)$ multipliziert und über $Q_{w}$ integriert. Die Randbedingung (15c) wird unter Verwendung des Divergenztheorems [22] berücksichtigt, wodurch sich die schwache Formulierung

$$
\begin{array}{r}
\int_{\mathrm{Q}_{\mathrm{w}}}\left(\rho C_{\mathrm{p}} \partial_{t} T \Phi+(k \nabla T) \cdot(\nabla \Phi)-\frac{\sigma \omega^{2}}{2}|A|^{2} \Phi\right) \mathrm{d} x \mathrm{~d} t \\
=\int_{\Sigma_{\mathrm{w}}}\left(\alpha\left(T_{\mathrm{a}}-T\right)+\epsilon \sigma_{\mathrm{SB}}\left(T_{\mathrm{a}}^{4}-T^{4}\right)\right) \Phi \mathrm{d} x \mathrm{~d} t \\
\forall \Phi \in W\left(0, t_{\mathrm{f}}\right)
\end{array}
$$

ergibt. Ein geeigneter Lösungsraum ist durch

$$
\begin{aligned}
W\left(0, t_{\mathrm{f}}\right):=\left\{T \in L^{2}\left(0, t_{\mathrm{f}} ; H^{1}\left(\Omega_{\mathrm{w}}\right)\right) \mid\right. \\
\\
\left.\partial_{t} T \in L^{2}\left(0, t_{\mathrm{f}} ; H^{1}\left(\Omega_{\mathrm{w}}\right)^{*}\right)\right\}
\end{aligned}
$$

mit dem Sobolev-Raum $H^{1}\left(\Omega_{\mathrm{w}}\right)$, dessen Dualraum $H^{1}\left(\Omega_{\mathrm{w}}\right)^{*}$ sowie dem Raum der quadratisch integrierbaren Funktionen $L^{2}(\cdot)$ gegeben $[2,24]$.

Nach gleicher Vorgehensweise ergibt sich die schwache Formulierung des Teilsystems (15e)-(15f) zu

$$
\begin{aligned}
\int_{Q}\left(\left(\mu^{-1} \operatorname{curl} A\right)\right. & \cdot(\operatorname{curl} \Lambda)+j \omega \sigma A \cdot \Lambda) \mathrm{d} x \mathrm{~d} t \\
= & \int_{\mathrm{Q}} J_{\mathrm{c}}(u) \cdot \Lambda \mathrm{d} x \mathrm{~d} t \quad \forall \Lambda \in X .
\end{aligned}
$$

Aufgrund der quasistationären Betrachtung des elektromagnetischen Teilsystems ist hierbei die Testfunktion $\Lambda:=\Lambda(x)$ zeitunabhängig. Zur Berücksichtigung der Randbedingung (15f) wird der Integralsatz

$$
\int_{\Omega} A \cdot(\operatorname{curl} \lambda)-(\operatorname{curl} A) \cdot \lambda \mathrm{d} x=\int_{\partial \Omega}(A \times \vec{n}) \cdot \lambda \mathrm{d} x
$$

verwendet [28]. Der zur schwachen Formulierung des elektromagnetischen Teilsystems zugehörige Lösungsraum ist durch

$$
\begin{aligned}
X:=\{A \in H(\operatorname{curl} ; \Omega) \cap H(\operatorname{div} ; \Omega) \mid \\
\nabla \cdot A=0 \text { in } \Omega, A \times \vec{n}=0 \text { auf } \partial \Omega\}
\end{aligned}
$$

mit den Räumen

$$
\begin{aligned}
& H(\operatorname{curl} ; \Omega):=\left\{A \in L^{2}(\Omega)^{3} \mid \operatorname{curl} A \in L^{2}(\Omega)^{3}\right\} \\
& H(\operatorname{div} ; \Omega):=\left\{A \in L^{2}(\Omega)^{3} \mid \nabla \cdot A \in L^{2}(\Omega)\right\}
\end{aligned}
$$

definiert [17, 24].

Für die numerische Lösung der Systemdynamik der induktiven Heizvorgänge können die schwachen Formulierungen (16) und (18) mit $(T, A) \in W\left(0, t_{\mathrm{f}}\right) \times X$ verwendet werden. Wie im folgenden Abschnitt gezeigt wird, ermöglichen diese zudem eine kompakte Herleitung der Optimalitätsbedingungen des betrachteten Optimierungsproblem (15).

\subsubsection{Anwendung der formalen Lagrange-Technik}

Eine aus der Literatur bekannte Methode zur Formulierung der Optimalitätsbedingungen von verteiltparametrischen Optimierungsproblemen, die sich auch für komplexere Probleme eignet, ist die formale LagrangeTechnik [23]. Die Bezeichnung „formal“ weist dabei auf die Vernachlässigung einer mathematisch strengen Definition der Lagrange-Multiplikatoren sowie der Funktionenräume der entsprechenden adjungierten Zustände hin.

Angewendet auf die betrachtete Problemstellung (15) wird zunächst die Lagrange-Funktion $\mathcal{L}:=$ $\mathcal{L}(T, A, u, p, \lambda)$ definiert. Hierfür werden die differentiellen Nebenbedingungen (15b)-(15c) sowie (15e)-(15f) mit den Lagrange-Multiplikatoren $p:=p(x, t)$ und $\lambda:=\lambda(x)$ integrativ über $Q_{w}$ bzw. $Q$ an das Gütefunktional (15a) gekoppelt. Unter Verwendung der schwachen Formulierungen (16) und (18) kann gezeigt werden, dass hierbei die Lagrange-Funktion

$$
\begin{aligned}
\mathcal{L} & =V\left(T\left(\cdot, t_{\mathrm{f}}\right)\right)+\int_{0}^{t_{\mathrm{f}}} l(T, u) \mathrm{d} t \\
+ & \int_{Q_{\mathrm{w}}}\left(\rho C_{\mathrm{p}} \partial_{t} T p+(k \nabla T) \cdot(\nabla p)-\frac{\sigma \omega^{2}}{2}|A|^{2} p\right) \mathrm{d} x \mathrm{~d} t \\
& -\int_{\Sigma_{\mathrm{w}}}\left(\alpha\left(T_{\mathrm{a}}-T\right)+\epsilon \sigma_{\mathrm{SB}}\left(T_{\mathrm{a}}^{4}-T^{4}\right)\right) p \mathrm{~d} x \mathrm{~d} t
\end{aligned}
$$




$$
+\int_{Q}\left(\left(\mu^{-1} \operatorname{curl} A\right) \cdot(\operatorname{curl} \lambda)+\left(j \omega \sigma A-J_{\mathrm{c}}(u)\right) \cdot \lambda\right) \mathrm{d} x \mathrm{~d} t
$$

resultiert, wobei die Testfunktionen $\Phi$ und $\Lambda$ durch die Lagrange-Multiplikatoren $p$ und $\lambda$ ersetzt werden.

Für eine optimale Lösung $y^{*}:=\left(T^{*}, A^{*}, u^{*}, p^{*}, \lambda^{*}\right)$ werden im Folgenden die Optimalitätsbedingungen erster Ordnung hergeleitet. Das zur thermischen Problemstellung gehörige adjungierte System kann dabei über die Betrachtung der partiellen Ableitung

$$
\begin{gathered}
\left.\frac{\partial \mathcal{L}}{\partial T}\right|_{y=y^{*}} h=\int_{\Omega_{\mathrm{w}}} q_{1} \zeta(x)\left(T^{*}\left(\cdot, t_{\mathrm{f}}\right)-T_{\mathrm{d}}\right) h \mathrm{~d} x \\
+\int_{\mathrm{Q}_{\mathrm{w}}}\left(q_{2} \zeta(x)\left(T^{*}-T_{\mathrm{d}}\right) h+q_{3} \max \left(0, T^{*}-T^{+}\right) h\right. \\
\left.+\rho C_{\mathrm{p}} p^{*} \partial_{t} h+\left(\nabla p^{*}\right) \cdot(k \nabla h)\right) \mathrm{d} x \mathrm{~d} t \\
+\int_{\Sigma_{\mathrm{w}}} \alpha p^{*} h+4 \epsilon \sigma_{\mathrm{SB}} T^{* 3} p^{*} h \mathrm{~d} x \mathrm{~d} t
\end{gathered}
$$

mit den zulässigen Richtungen $h:=h(x, t)$ bestimmt werden.

Um die Optimalitätsbedingung erster Ordnung $\left.\frac{\partial \mathcal{L}}{\partial T}\right|_{y=y^{*}} h \stackrel{!}{=} 0 \quad \forall h$ auswerten $\mathrm{zu}$ können, müssen die ableitungsbehafteten Anteile der Richtungen $h$ auf den Zustand $T$ verlagert werden. Hierfür wird die Formel der partiellen Integration auf den vierten Anteil der rechten Seite von (23) angewendet, d.h.,

$$
\begin{aligned}
& \int_{Q_{\mathrm{w}}} \rho C_{\mathrm{p}} p^{*} \partial_{t} h \mathrm{~d} x \mathrm{~d} t= \\
& \left.\quad \int_{\Omega_{\mathrm{w}}} \rho C_{\mathrm{p}} p^{*} h\right|_{t=0} ^{t=t_{\mathrm{f}}} \mathrm{d} x-\int_{Q_{\mathrm{w}}} \rho C_{\mathrm{p}} \partial_{t} p^{*} h \mathrm{~d} x \mathrm{~d} t .
\end{aligned}
$$

Des Weiteren wird für den fünften Anteil der rechten Seite von (23) die erste Green'sche Formel

$$
\begin{aligned}
& \int_{Q_{\mathrm{w}}}\left(\nabla p^{*}\right) \cdot(k \nabla h) \mathrm{d} x \mathrm{~d} t= \\
& -\int_{Q_{\mathrm{w}}} \nabla \cdot\left(k \nabla p^{*}\right) h \mathrm{~d} x \mathrm{~d} t+\int_{\Sigma_{\mathrm{w}}} \vec{n} \cdot\left(k \nabla p^{*}\right) h \mathrm{~d} x \mathrm{~d} t
\end{aligned}
$$

benötigt. Da $\left.h\right|_{t=0}$ aufgrund der gegebenen Anfangsbedingung (15d) identisch Null sein muss, erhält man

$$
\left.\frac{\partial \mathcal{L}}{\partial T}\right|_{y=y^{*}} h=\int_{\Omega_{\mathrm{w}}}\left[q_{1} \zeta(x)\left(T^{*}\left(\cdot, t_{\mathrm{f}}\right)-T_{\mathrm{d}}\right)+\rho C_{\mathrm{p}} p^{*}\left(\cdot, t_{\mathrm{f}}\right)\right] h \mathrm{~d} x
$$

$$
\begin{gathered}
-\int_{Q_{\mathrm{w}}}\left[\rho C_{\mathrm{p}} \partial_{t} p^{*}+\nabla \cdot\left(k \nabla p^{*}\right)-q_{2} \zeta(x)\left(T^{*}-T_{\mathrm{d}}\right)\right. \\
\left.-q_{3} \max \left(0, T^{*}-T^{+}\right)\right] h \mathrm{~d} x \mathrm{~d} t \\
+\int_{\Sigma_{\mathrm{w}}}\left[\vec{n} \cdot\left(k \nabla p^{*}\right)+\left(\alpha+4 \epsilon \sigma_{\mathrm{SB}} T^{* 3}\right) p^{*}\right] h \mathrm{~d} x \mathrm{~d} t .
\end{gathered}
$$

Die Auswertung der Optimalitätsbedingung erster Ordnung $\left.\frac{\partial \mathcal{L}}{\partial T}\right|_{y=y^{*}} h \stackrel{!}{=} 0 \forall h$ führt somit auf das zur thermischen Problemstellung (15b)-(15d) zugehörige adjungierte System

$$
\begin{array}{ll}
\rho C_{\mathrm{p}} \partial_{t} p+\nabla \cdot(k \nabla p)=q_{2} \zeta(x)\left(T-T_{\mathrm{d}}\right) & \\
\quad+q_{3} \max \left(0, T-T^{+}\right) & \text {in } \mathrm{Q}_{\mathrm{w}} \\
\vec{n} \cdot(k \nabla p)=-\left(\alpha+4 \epsilon \sigma_{\mathrm{SB}} T^{3}\right) p & \text { auf } \Sigma_{\mathrm{w}} \\
\rho C_{\mathrm{p}} p\left(\cdot, t_{\mathrm{f}}\right)=-q_{1} \zeta(x)\left(T\left(\cdot, t_{\mathrm{f}}\right)-T_{\mathrm{d}}\right) & \text { in } \Omega_{\mathrm{w}}
\end{array}
$$

in (starker) pDGl-Formulierung. Auf eine Kennzeichnung der optimalen Größen wird hierbei verzichtet.

Für die Herleitung des adjungierten Systems der elektromagnetischen Problemstellung wird die partielle Ableitung

$$
\begin{aligned}
& \left.\frac{\partial \mathcal{L}}{\partial A}\right|_{y=y^{*}} h=-\int_{Q_{\mathrm{w}}} \sigma \omega^{2}\left|A^{*}\right| p^{*} \cdot h \mathrm{~d} x \mathrm{~d} t \\
& +\int_{Q}\left(\left(\operatorname{curl} \lambda^{*}\right) \cdot\left(\mu^{-1} \operatorname{curl} h\right)+j \omega \sigma \lambda^{*} \cdot h\right) \mathrm{d} x \mathrm{~d} t
\end{aligned}
$$

betrachtet. Der ableitungsbehaftete Anteil der zulässigen Richtungen $h:=h(x)$ kann mit Hilfe des Integralsatzes (19) auf den Zustand $A$ verlagert werden

$$
\begin{aligned}
& \left.\frac{\partial \mathcal{L}}{\partial A}\right|_{y=y^{*}} h=-\int_{Q_{\mathrm{w}}} \sigma \omega^{2}\left|A^{*}\right| p^{*} \cdot h \mathrm{~d} x \mathrm{~d} t \\
& \quad+\int_{Q}\left[\operatorname{curl}\left(\mu^{-1} \operatorname{curl} \lambda^{*}\right)+j \omega \sigma \lambda^{*}\right] \cdot h \mathrm{~d} x \mathrm{~d} t .
\end{aligned}
$$

Die Auswertung der Optimalitätsbedingung erster Ordnung $\left.\frac{\partial \mathcal{L}}{\partial A}\right|_{y=y^{*}} h \stackrel{!}{=} 0 \forall h$ führt somit auf das zur elektromagnetischen Problemstellung (15e)-(15f) zugehörige adjungierte System

$$
\begin{array}{ll}
\operatorname{curl}\left(\mu^{-1} \operatorname{curl} \lambda\right)+j \omega \sigma \lambda=\sigma \omega^{2}|A| p & \text { in } Q_{\mathrm{w}} \\
\operatorname{curl}\left(\mu^{-1} \operatorname{curl} \lambda\right)+j \omega \sigma \lambda=0 & \text { in } Q \backslash Q_{\mathrm{w}} \\
\lambda \times \vec{n}=0 & \text { auf } \Sigma
\end{array}
$$

in (starker) pDGl-Formulierung.

Eine Formulierung der Optimalitätsbedingung erster Ordnung hinsichtlich der Stellgröße $u$ kann durch die Be- 
trachtung der Variationsungleichung

$$
\left.\frac{\partial \mathcal{L}}{\partial u}\right|_{y=y^{*}}\left(u-u^{*}\right) \stackrel{!}{\geq} 0 \quad \forall u \in \mathcal{U}_{\mathrm{ad}}
$$

erreicht werden. Durch die Definition des reduzierten Gradienten $g(t)$ mit Hilfe des Satzes von Riesz, siehe z.B. [23],

$$
g(t):=\left.\frac{\partial \mathcal{L}}{\partial u}\right|_{y=y^{*}}=q_{4} u^{*}-\int_{\Omega_{c}} \frac{N_{c}}{A_{c}} \vec{e}_{c} \cdot \lambda^{*} \mathrm{~d} x
$$

kann für eine optimale Stellgröße $u^{*}$ die Bedingung

$$
g(t)\left\{\begin{array}{cl}
\stackrel{!}{>} 0 & \text { falls } u^{*}(t)=u^{-} \\
\stackrel{!}{=} 0 & \text { falls } u^{*}(t) \in\left(u^{-}, u^{+}\right) \quad \forall t \in\left(0, t_{\mathrm{f}}\right) \\
\vdots &
\end{array}\right.
$$

formuliert werden.

Als Ergebnis der formalen Lagrange-Technik stehen neben der Bedingung (33) für die optimale Stellgröße die adjungierten Systeme (27) und (30) für eine numerische Lösung des Optimierungsproblems (15) zur Verfügung. Auf Basis dieser Optimalitätsbedingungen wird im folgenden Abschnitt mit dem Gradientenverfahren ein Optimierungsverfahren vorgestellt, um auch komplexere Problemstellungen wie das des induktiven Heizens handhaben zu können.

\section{Numerische Lösung}

Die mit einem Optimalsteuerungsentwurf für MultiphysikProbleme verbundene Lösung des Optimalitätssystems erfordert im Allgemeinen einen hohen Implementierungsaufwand. Im Falle des induktiven Heizens wird dieser im Wesentlichen durch die Lösung der kanonischen Gleichungen (5), (8), (27) und (30) verursacht. Im Folgenden wird näher ausgeführt, wie der numerische Aufwand maßgeblich auf FEM-Software ausgelagert werden kann.

\subsection{Gradientenverfahren}

Das Gradientenverfahren nutzt neben der Eigenschaft der unbeschränkten Zustände des Optimierungsproblems (15) die strukturelle Eigenschaft der Optimalitätsbedingungen aus, dass durch die Randbedingung (27c) der adjungierte Zustand $p$ zum Endzeitpunkt $t_{\mathrm{f}}$ bekannt ist. Dies erlaubt eine sequentielle numerische Lösung der kanonischen Gleichungen (8) und (27), wobei im Falle der betrachteten induktiven Heizvorgänge noch die unterlagerten algebraischen Beschränkungen (5) bzw. (30) berücksichtigt werden müssen.

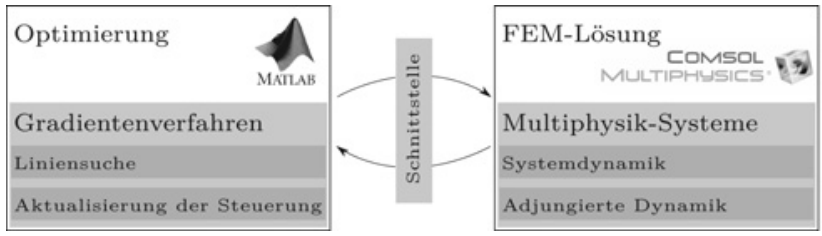

Abbildung 2: Softwareumgebung für die numerische Lösung von Multiphysik-Problemen.

Ausgehend von einer initialen Steuertrajektorie $u^{(0)}(t)$ umfasst eine Iteration $j$ des Gradientenverfahrens die folgenden Schritte:

- Berechnung der Profile $\left(T^{(j)}, A^{(j)}\right)$ mit der Steuerung $u^{(j)}$ durch Vorwärtssimulation von (5) und (8),

- Berechnung der Profile $\left(p^{(j)}, \lambda^{(j)}\right)$ mit $\left(T^{(j)}, A^{(j)}\right)$ durch Rückwärtssimulation von (27) und (30),

- Gradientenauswertung $g(t)$ gemäß (32),

- Aktualisierung der Steuerung $u^{(j+1)}=\psi\left(u^{(j)}-\alpha^{(j)} g^{(j)}\right)$.

Im Hinblick auf (32) und (33) wird zur Aktualisierung der Steuertrajektorie $u^{(j+1)}$ für die nächste Iteration $j+1$ die Sättigungsfunktion

$$
\psi(u)= \begin{cases}u^{-} & \text {falls } u<u^{-} \\ u^{+} & \text {falls } u>u^{+} \\ u & \text { sonst }\end{cases}
$$

zur Einhaltung der Eingangsbeschränkungen (9) verwendet. Für die Bestimmung der Schrittweite $\alpha^{(j)}$ kann ein Liniensuchproblem formuliert werden, das in der Regel approximativ gelöst wird [8].

\subsection{Implementierung}

Der verwendete FOTD-Ansatz mit den Optimalitätsbedingungen in Form von pDGln in Kombination mit dem Gradientenverfahren ermöglichen es, den mit MultiphysikProblemen einhergehenden numerischen Aufwand auf FEM-Software auszulagern. Erste Ergebnisse am Beispiel induktiver Aufheizvorgänge auf einfacheren Ortsgeometrien werden hierzu in [20] präsentiert.

Die Grundidee besteht darin, die Koordinierung des Gradientenverfahrens in MATLAB zu realisieren, während die numerische Lösung der kanonischen Gleichungen (5), (8), (27) und (30) auf COMSOL Multiphysics ausgelagert wird, vergleiche Abbildung 2. Eine geeignete Schnittstelle für die Interaktion zwischen MATLAB und COMSOL MulTiphysics ist mit dem COMSOL MulTiPHYSICS-Modul LiveLink FOR MATLAB gegeben. Dieses ermöglicht neben dem Starten der Vorwärts- und Rück- 
wärtssimulationen der kanonischen Gleichungen auch die Nachbearbeitung und Aufbereitung der numerischen Ergebnisse.

Für die numerische Lösung der kanonischen Gleichungen werden die schwachen Formulierungen der Systemdynamik (16) und (18) mit Hilfe des PhysicsBuilDER-Moduls in COMSOL MULTIPHYSICS implementiert. Die schwachen Formulierungen der adjungierten Dynamik (27) und (30) werden mit Hilfe der in Abschnitt 3.2.1 angegebenen Integralsätze bestimmt und ebenfalls in COMSOL MulTiPhysics implementiert.

Durch den vorgestellten Ansatz kann für die dynamische Optimierung der induktiven Heizvorgänge sowohl auf spezialisierte Algorithmen zur Ortsdiskretisierung komplexerer Geometrien als auch für die numerische Lösung der kanonischen Gleichungen zurückgegriffen werden. Infolgedessen wird eine flexible Abbildung verschiedener Aufbauten induktiver Heizvorgänge ermöglicht. Dies trifft sowohl auf die Geometrie des zu erhitzenden Werkstücks als auch auf die Geometrie und Anordnung der Spule zu, wobei Letzteres eine angepasste Formulierung der Richtung des Stromflusses (6) erfordert. Somit können zahlreiche praktische Anwendungsbeispiele induktiver Heizvorgänge abgebildet werden, siehe zum Beispiel [21].

\section{Simulationsergebnisse}

Basierend auf dem vorgestellten Ansatz zur dynamischen Optimierung von Multiphysik-Problemen werden induktive Aufheiz- und Oberflächenhärtungsvorgänge für ein Zahnrad in einer Simulationsumgebung betrachtet. Der für beide Problemstellungen identische Aufbau wird im folgenden Abschnitt beschrieben. Anschließend werden die jeweiligen Gütefunktionale spezifiziert sowie die damit erzielten Simulationsergebnisse vorgestellt.

\subsection{Aufbau}

Der im Folgenden betrachtete Aufbau zum induktiven Aufheizen eines Zahnrads aus Stahl ist unter Berücksichtigung vorhandener Symmetrien in Abbildung 3 zu sehen. Die vollständige Geometrie des Zahnrads mit 15 Zähnen weist dabei die Höhe $h_{\mathrm{G}}=8 \mathrm{~mm}$ sowie einen Fußkreisdurchmesser von $r_{\mathrm{G}_{1}}=27 \mathrm{~mm}$ und einen Kopfkreisdurchmesser von $r_{\mathrm{G}_{2}}=38 \mathrm{~mm}$ auf. Das Ortsgebiet für die um die $x_{3}$-Achse rotationssymmetrische Spule aus Kupfer mit dem Radius $r_{c}=4 \mathrm{~mm}$ umfasst insgesamt $N_{c}=100$ Windungen. Die umgebende Luft wird mit einem entsprechen-

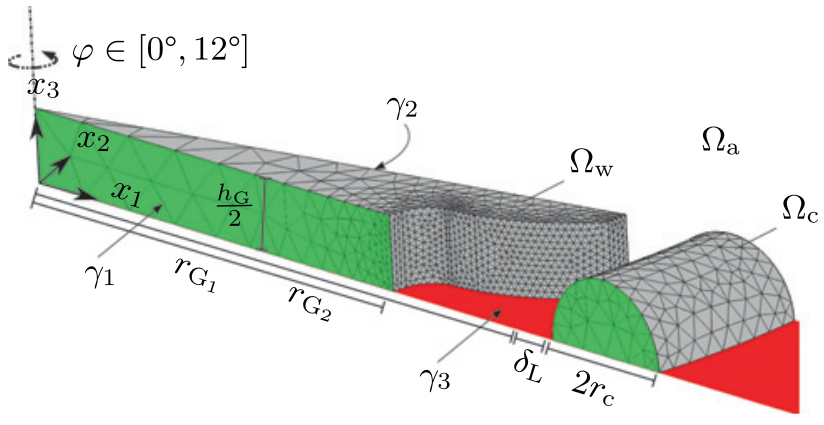

Abbildung 3: Aufbau zum induktiven Aufheizen eines Zahnrads unter Ausnutzung von Symmetrieebenen (grün und rot).

Tabelle 1: Materialparameter der induktiven Aufheiz- und Oberflächenhärtungsvorgänge.

\begin{tabular}{lrrrr}
\hline & Zahnrad & Spule & \multicolumn{2}{c}{ Luft } \\
& $\Omega_{\mathrm{w}}$ & $\Omega_{\mathrm{c}}$ & $\Omega_{\mathrm{a}}$ & \\
\hline$\mu_{\mathrm{r}}$ & $10^{2}$ & 1 & 1 & {$[-]$} \\
$\sigma$ & $4.03 \cdot 10^{6}$ & $10^{-3}$ & $10^{1}$ & {$\left[\mathrm{~S} \mathrm{~m}^{-1}\right]$} \\
$\rho$ & $7.85 \cdot 10^{3}$ & - & - & {$\left[\mathrm{kg} \mathrm{m}^{-3}\right]$} \\
$C_{\mathrm{p}}$ & 475 & - & - & {$\left[\mathrm{J} \mathrm{kg}^{-1} \mathrm{~K}^{-1}\right]$} \\
$k$ & 44.5 & - & - & {$\left[\mathrm{W} \mathrm{m}^{-1} \mathrm{~K}^{-1}\right]$} \\
$\alpha$ & 1.54 & - & - & {$\left[\mathrm{W} \mathrm{m}^{-2} \mathrm{~K}^{-1}\right]$} \\
$\epsilon$ & 0.7 & - & - &
\end{tabular}

den Zylinderabschnitt mit dem Radius $r_{\mathrm{L}}=0.25 \mathrm{~m}$ und der Höhe $h_{\mathrm{L}}=0.2 \mathrm{~m}$ modelliert, wobei der Luftspalt zwischen Zahnrad und Spule im Minimum $\delta_{\mathrm{L}}=1.5 \mathrm{~mm}$ beträgt.

Die für die Simulationen verwendeten Materialparameter der elektromagnetischen und thermischen Problemstellung (5) und (8) sind in Tabelle 1 zusammengefasst. Aus numerischen Gründen wird für die Gebiete $\Omega_{\mathrm{c}}$ und $\Omega_{\mathrm{a}}$ eine elektrische Leitfähigkeit ungleich Null angenommen.

Durch die Berücksichtigung der Symmetrieebenen kann das Volumen der Problemstellung um den Faktor 60 reduziert werden, was zu einem signifikant geringerem numerischen Aufwand für die Lösung der kanonischen Gleichungen (5), (8), (27) und (30) führt. Ergänzend zu den bisher beschriebenen Randbedingungen muss für das elektromagnetische Problem (5) eine magnetische Isolierung mit Hilfe der Randbedingung

$$
A \times \vec{n}=0 \quad \text { auf } \gamma_{1,2} \times\left(0, t_{\mathrm{f}}\right)
$$

an der in Abbildung 3 grün markierten Symmetrieebene $\gamma_{1}:=\left\{x \in \Omega \mid x_{2}=0\right\}$ sowie an der um den Winkel $\varphi=$ $12^{\circ}$ gedrehten Ebene $\gamma_{2}$ betrachtet werden. Auf der dritten Symmetrieebene $\gamma_{3}=\left\{x \in \Omega \mid x_{3}=0\right\}$ muss hingegen eine perfekte magnetische Leitung

$$
\left(\mu^{-1} \operatorname{curl} A\right) \times \vec{n}=0 \quad \text { auf } \gamma_{3} \times\left(0, t_{\mathrm{f}}\right)
$$


berücksichtigt werden, vergleiche hierzu die rot markierte Ebene in Abbildung 3. Die Randbedingungen der Wärmeleitungsgleichung (8) erweitern sich zudem um

$$
\vec{n} \cdot(k \nabla T)=0 \quad \text { auf }\left(\gamma_{1,2} \cup \gamma_{3}\right) \times\left(0, t_{\mathrm{f}}\right),
$$

was einem verschwindenden Wärmefluss entspricht.

\subsection{Induktiver Aufheizvorgang}

Eine geeignete Wahl für die Realisierung eines Aufheizvorgangs von $T_{0}=20^{\circ} \mathrm{C}$ auf das örtlich konstante Temperaturprofil $T_{\mathrm{d}}=500{ }^{\circ} \mathrm{C}$ in $t_{\mathrm{f}}=40 \mathrm{~s}$ ist durch das Gütefunktional (11) mit der Funktion $\zeta(x)$ gemäß (12) gegeben. Für die Zustandsbeschränkung (10) wird die Schranke $T^{+}=$ $580^{\circ} \mathrm{C}$ betrachtet, während die Stromstärke der Spule mit der Kreisfrequenz $\omega=1 \mathrm{kHz}$ auf das Intervall [0 A, $200 \mathrm{~A}$ ] beschränkt wird. Die Gewichtungsfaktoren des Gütefunktionals werden zu $q_{i}=\left\{10^{2}, 10^{6}, 10^{9}, 10^{-2}\right\}$ gewählt. $^{1}$

Abbildung 4 zeigt die Ergebnisse des beschriebenen Szenarios in Form des Verlaufs des Spulenstroms $u(t)$ sowie der zugehörigen zeitlichen Verläufe der minimalen Temperatur $T_{\min }$, der durchschnittlichen Temperatur $T_{\text {avg }}$ sowie der maximalen Temperatur $T_{\max }$ während des induktiven Aufheizvorgangs. Das leichte Überschreiten der maximal zulässigen Temperatur $T^{+}=580{ }^{\circ} \mathrm{C}$ zwischen $7 \mathrm{~s}$ und $20 \mathrm{~s}$ ist auf die lediglich indirekt berücksichtigte $\mathrm{Zu}$ standsbeschränkung (10) mit Hilfe einer äußeren Straffunktion zurückzuführen, vergleiche (11). Durch eine Erhöhung des Gewichtungsfaktors $q_{3}$ kann die leichte Überschreitung der maximalen Temperatur $T_{\max }$ weiter reduziert werden.

Die geringe Leitfähigkeit von Stahl in Kombination mit der zu berücksichtigenden Zustandsbeschränkung erfordert eine relativ lange Endzeit von $t_{\mathrm{f}}=40 \mathrm{~s}$, was sich auch in dem Stellgrößenverlauf $u(t)$ und den Verläufen der Werkstücktemperaturen in Abbildung 4 widerspiegelt. $\mathrm{Zu}$ Beginn des induktiven Aufheizvorgangs wird daher unter Beachtung der Systemdynamik sowie der zu berücksichtigenden Zustandsbeschränkung mit maximal zulässiger Leistung aufgeheizt. Anschließend folgt bis ungefähr $t=22 \mathrm{~s}$ eine Phase, in welcher dem System gerade soviel Energie zugeführt wird, wie es die Zustandsbeschränkung erlaubt. Im restlichen Zeitintervall wird dann nahezu keine weitere Energie mehr zugeführt und die Wärmelei-

1 Es sei angemerkt, dass der vierte Anteil im Gütefunktional (11) kein Ortsintegral enthält und somit aufgrund des geringen Volumen des Zahnrads geringer gewichtet wird.
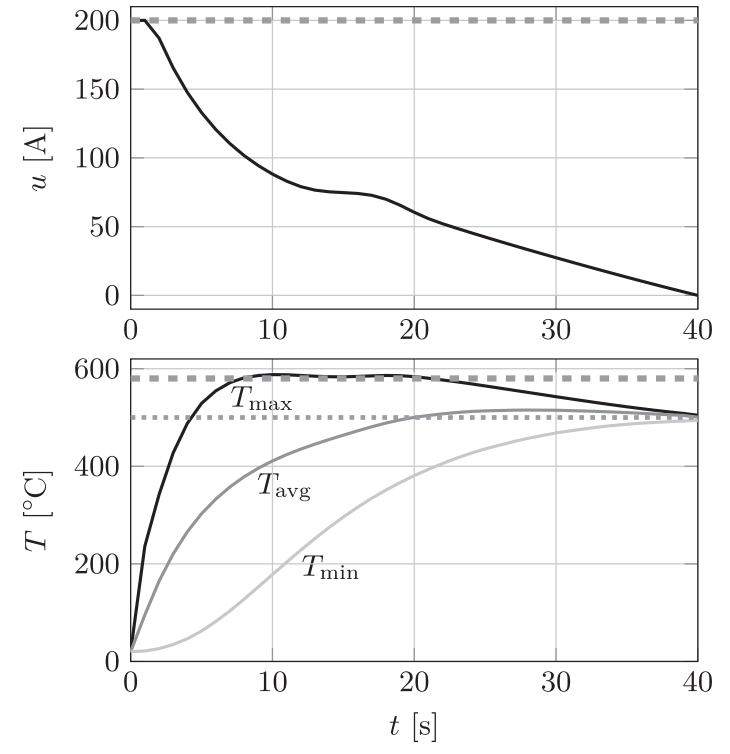

Abbildung 4: Spulenstrom $u(t)$ sowie Verläufe der minimalen, der durchschnittlichen und der maximalen Werkstücktemperatur während des induktiven Aufheizvorgangs.

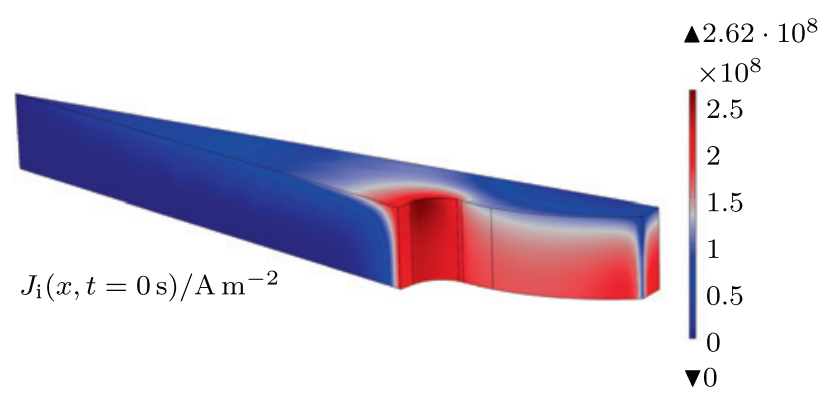

Abbildung 5: Induzierte Ströme $J_{\mathrm{i}}$ für die Problemstellung des induktiven Aufheizens zum Zeitpunkt $t=0 \mathrm{~s}$.

tung führt zum Erreichen der gewünschten Solltempera$\operatorname{tur} T_{\mathrm{d}}=500{ }^{\circ} \mathrm{C}$.

Wie in Abbildung $5 \mathrm{zu}$ sehen, führen neben dem SkinEffekt die auftretenden End- und Edge-Effekte zu einer auch entlang der Randschicht stark unterschiedlichen Verteilung der induzierten Ströme $J_{\mathrm{i}}=j \omega \sigma A$. Die Herausforderung für den Optimalsteuerungsentwurf beruht somit auch auf der Tatsache, dass sich der Einfluss der Stellgröße zum Großteil auf den Randbereich beschränkt.

Der Bereich mit der höchsten induzierten Leistung befindet sich wie in Abbildung 5 zu erkennen ist am Zahnfuß und kann auf die End- und Edge-Effekte zurückgeführt werden. Allerdings sorgt die vorliegende Ortsgeometrie in Kombination mit der Wärmeleitung dazu, dass die maximale Werkstücktemperatur $T_{\max }=584.6^{\circ} \mathrm{C}$ zum Zeitpunkt $t=10.5 \mathrm{~s}$ am Zahnkopf erreicht wird. Für die induktiven Aufheizvorgänge kann festgehalten werden, 
dass durch die numerische Lösung des dynamischen Optimierungsproblems (15) unter Beachtung der formulierten Eingangs- und Zustandsbeschränkungen ein gutes Systemverhalten erzielt wird.

\subsection{Induktive Oberflächenhärtung}

In einem zweiten Szenario wird gezeigt, dass unter Verwendung des vorherigen Optimierungsproblems mit der speziellen Wahl (13) für die Funktion $\zeta(x)$ auch Oberflächenhärtungsvorgänge abgebildet werden können. Hierfür soll das auf $T_{0}=500{ }^{\circ} \mathrm{C}$ vorgeheizte Zahnrad am Rand bis zu einer Tiefe von $\delta_{\mathrm{p}}=2 \mathrm{~mm}$ auf $T_{\mathrm{d}}=750{ }^{\circ} \mathrm{C}$ erhitzt werden. Für diesen Heizvorgang mit der angepassten Horizontlänge $t_{\mathrm{f}}=8 \mathrm{~s}$ wird die Zustandsbeschränkung $\mathrm{zu} T^{+}=830^{\circ} \mathrm{C}$ gewählt und der Spulenstrom auf das Intervall $[0 \mathrm{~A}, 30 \mathrm{~A}$ ] bei einer Kreisfrequenz von $\omega=50 \mathrm{kHz}$ beschränkt. Die Gewichtungsfaktoren des Gütefunktionals werden zu $q_{i}=\left\{10^{6}, 10^{5}, 10^{9}, 10^{-2}\right\}$ abgeändert.

Der Skin-Effekt führt aufgrund der höher gewählten Kreisfrequenz $\omega=50 \mathrm{kHz}$ dazu, dass die End- und EdgeEffekte nicht mehr so stark ausgeprägt sind wie dies im Falle des vorherigen Aufheizvorgangs festzustellen war. Ein Vergleich der induzierten Ströme im Falle der Oberflächenhärtung aus Abbildung 6 mit der entsprechenden Stromverteilung des Aufheizvorgangs aus Abbildung 5 macht deutlich, dass jetzt auch im Bereich des Zahnkopfes mehr Ströme induziert werden. Diese angepasste Stromverteilung kann für die Oberflächenhärtung genutzt werden, da hierdurch ein homogenerer Wärmeeintrag entlang der Randschicht erzeugt wird.

Als Ergebnis der dynamischen Optimierung für die Oberflächenhärtung wird die Eingangstrajektorie $u(t)$ aus Abbildung 7 erzielt. Darüber hinaus sind dort die Verläufe der minimalen, der durchschnittlichen und der maximalen Temperatur $\tilde{T}_{\min }(t), \tilde{T}_{\text {avg }}(t)$ und $\tilde{T}_{\max }(t)$ im Bereich der zu härtenden Randschicht (14) zu sehen.

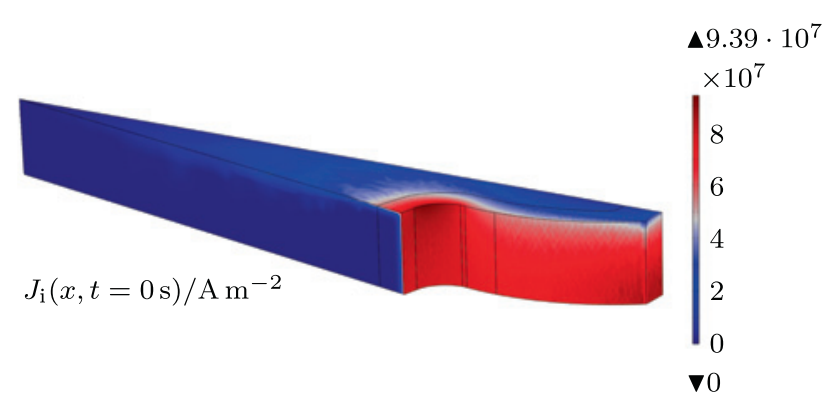

Abbildung 6: Induzierte Ströme $J_{\mathrm{i}}$ für die Problemstellung der Oberflächenhärtung zum Zeitpunkt $t=0 \mathrm{~s}$.

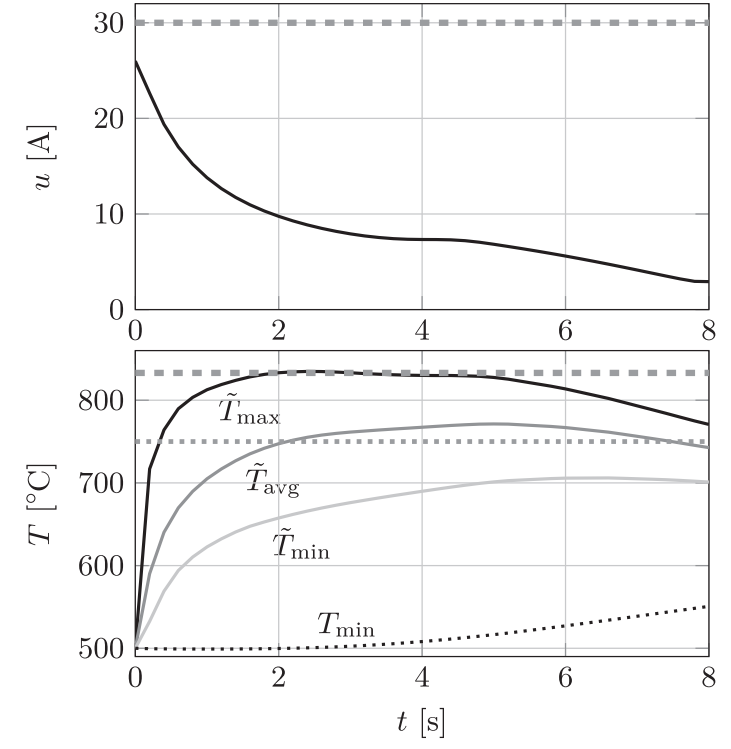

Abbildung 7: Spulenstrom $u(t)$ sowie Verläufe der minimalen, der durchschnittlichen und der maximalen Werkstücktemperatur in $\tilde{\Omega}_{\mathrm{w}}$ bzw. $\Omega_{\mathrm{w}}$ während der Oberflächenhärtung.

Wie bereits beim induktiven Aufheizvorgang ist auch bei der Oberflächenhärtung die Eingangstrajektorie $u(t)$ so ausgelegt, dass zu Beginn des Heizvorgangs genügend Energie über die induzierten Ströme in das Werkstück eingebracht wird. Die Einhaltung der Zustandsbeschränkung $\mathrm{T}^{+}=830^{\circ} \mathrm{C}$ wird hierbei geeignet berücksichtigt, was anhand des Verlaufs von $\tilde{T}_{\max }(t)$ in Abbildung 7 zu erkennen ist.

Da das Ziel der Oberflächenhärtung, d.h., die partielle Erwärmung der Randschicht, ein stark instationäres Problem darstellt und der Einfluss auf die Systemdynamik durch die Stellgröße $u(t)$ begrenzt ist, kann das gewünschte Sollprofil $T_{\mathrm{d}}$ nur näherungsweise erreicht werden. Am Ende des induktiven Heizvorgangs wird allerdings innerhalb des Gebiets $\tilde{\Omega}_{\mathrm{w}}$ ein relativ kleines Temperaturintervall angefahren, welches durch die charakteristischen Temperaturen $\tilde{T}_{\min }\left(t_{\mathrm{f}}\right)=702^{\circ} \mathrm{C}, \tilde{T}_{\text {avg }}\left(t_{\mathrm{f}}\right)=744^{\circ} \mathrm{C}$ und $\tilde{T}_{\max }\left(t_{\mathrm{f}}\right)=770{ }^{\circ} \mathrm{C}$ beschrieben ist.

Für die Martensitbildung ist ein anschließender rascher Abkühlvorgang des Werkstücks erforderlich, weshalb die inneren Bereiche des Werkstücks eine möglichst geringe Temperatur aufweisen sollten [21]. Ein guter Indikator für die zu erreichende Härte ist daher durch die minimale Werkstücktemperatur im gesamten Gebiet $\Omega_{\mathrm{w}}$, d.h. $T_{\min }(t)$, gegeben, dessen zeitlicher Verlauf ebenfalls in Abbildung 7 dargestellt ist. Der zwangsläufige Anstieg von $T_{\min }(t)$ mit zunehmender Heizdauer erfordert einen Kompromiss zwischen dem zu minimierenden Abstand 

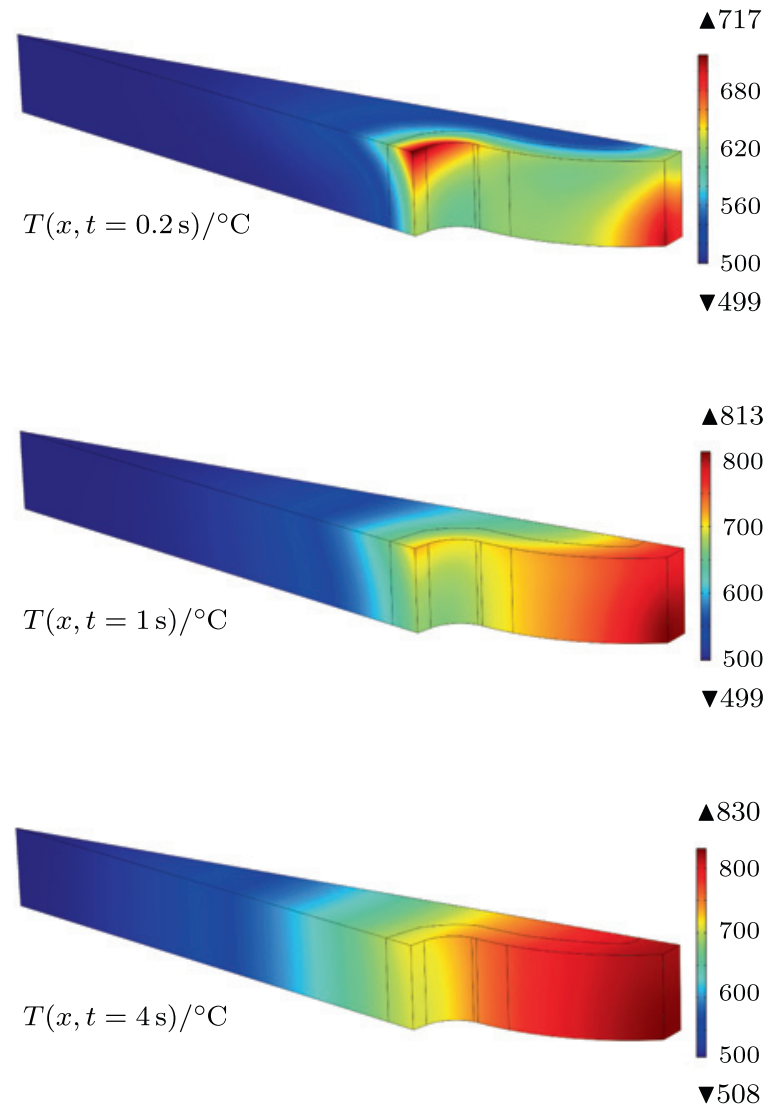
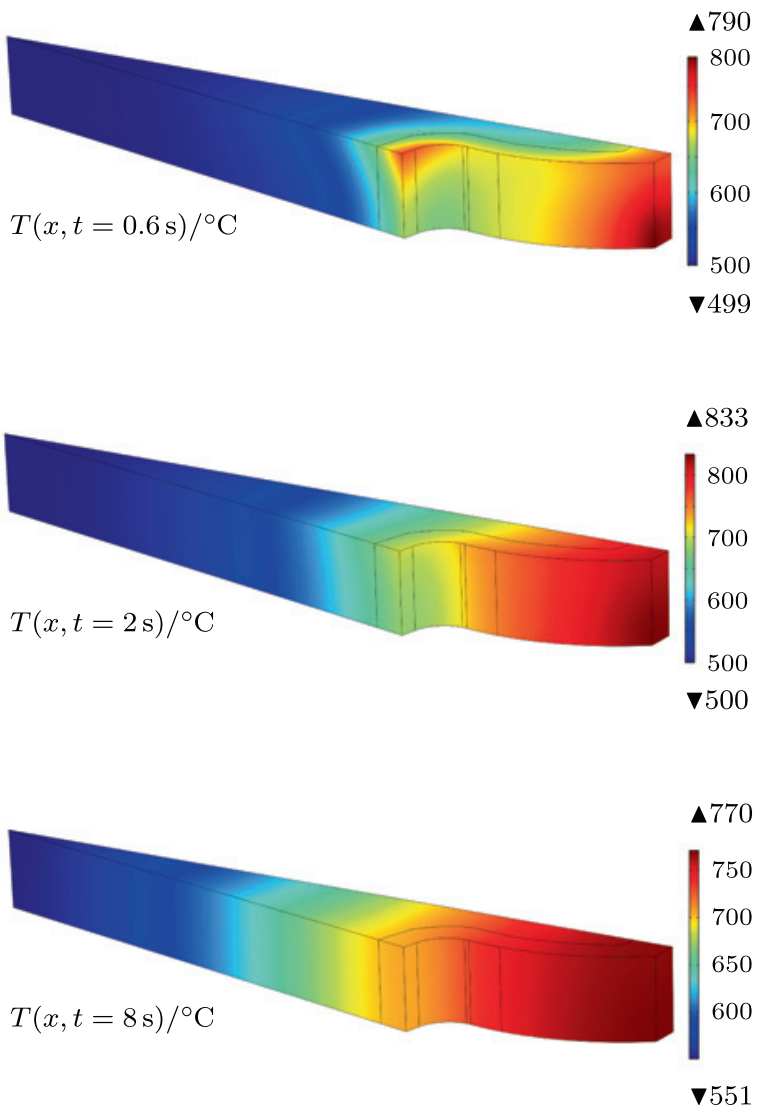

Abbildung 8: Temperaturprofile $T(x, t)$ des Zahnrads während der Oberflächenhärtung für $t \in\{0.2 \mathrm{~s}, 0.6 \mathrm{~s}, 1 \mathrm{~s}, 2 \mathrm{~s}, 4 \mathrm{~s}, 8 \mathrm{~s}\}$.

des Temperaturintervalls $\left[\tilde{T}_{\min }\left(t_{\mathrm{f}}\right), \tilde{T}_{\mathrm{avg}}\left(t_{\mathrm{f}}\right), \tilde{T}_{\max }\left(t_{\mathrm{f}}\right)\right]$ zum Sollprofil $T_{\mathrm{d}}$ und der Aufheizung der inneren Bereiche. In dem betrachteten Szenario wurde hierfür die Horizontlänge $t_{\mathrm{f}}=8 \mathrm{~s}$ gewählt.

Der beschriebene Oberflächenhärtungsvorgang ist anhand von Temperaturverläufen ausgewählter Zeitpunkte in Abbildung 8 dargestellt. Dabei kann anhand des Temperaturprofils $T(x, t)$ für den Zeitpunkt $t=0.2 \mathrm{~s}$ beobachtet werden, dass der Wärmeeintrag aufgrund des dominierenden Skin-Effekts überwiegend im erwünschten Gebiet $\tilde{\Omega}_{\mathrm{w}}$ erfolgt. Allerdings tendiert der Zahnkopf im weiteren Verlauf des Heizvorgangs aufgrund der ablaufenden Wärmeleitung in Kombination mit der vorliegenden Ortsgeometrie zu einer Überhitzung, was insbesondere zum Zeitpunkt $t=2 \mathrm{~s}$ erkannt werden kann.

Zum Zeitpunkt $t=2.4 \mathrm{~s}$ wird mit $T_{\max }=833.7^{\circ} \mathrm{C}$ die maximale Werkstücktemperatur während der Oberflächenhärtung erreicht. Insgesamt lässt sich somit festhalten, dass mit Hilfe des Optimierungsproblems (15) sowohl die Aufheizvorgänge als auch die Oberflächenhärtungsvorgänge realisiert werden können.

\subsection{Numerischer Aufwand}

Die numerische Lösung der kanonischen Gleichungen (5), (8), (27) und (30) im Rahmen des implementierten Gradientenverfahrens erfolgt in COMSOL MuLTiPHysics unter Verwendung eines BDF-Verfahrens. Um hierbei den Skin-Effekt sowie die beschriebenen End- und Edge-Effekte hinreichend genau auflösen zu können, wird vor allem am Randbereich des Zahnrads eine feine Ortsdiskretisierung benötigt.

Für die induktiven Aufheizvorgänge wurde das gesamte Ortsgebiet $\Omega$ mit $N=20.8 \cdot 10^{3}$ Tetraedern diskretisiert, wobei hiervon mit $N_{\mathrm{w}}=12.4 \cdot 10^{3}$ der Großteil der Elemente im Ortsgebiet des Werkstücks konzentriert ist. Einen Eindruck der Vernetzung für dieses erste Szenario vermittelt Abbildung 3. Um den stärker ausgeprägten SkinEffekt im Falle der Oberflächenhärtung hinreichend genau auflösen zu können, wurde für diese Problemstellung mit $N=25 \cdot 10^{3}$ bzw. mit $N_{\mathrm{w}}=14 \cdot 10^{3}$ eine feinere Ortsdiskretisierung gewählt.

Für das Szenario des induktiven Aufheizvorgangs zeigt sich der erforderliche Aufwand für die numerische Lösung des Optimierungsproblems (15) in $1.6 \cdot 10^{5}$ zu be- 
achtenden Freiheitsgraden für sowohl die Systemdynamik als auch für die adjungierte Dynamik. Im Falle der Oberflächenhärtung sind es $2 \cdot 10^{5}$ Freiheitsgrade. Eine Gradienteniteration benötigt dabei durchschnittlich $t_{\mathrm{CPU}}=38 \mathrm{~min}$ bzw. $t_{\mathrm{CPU}}=62 \mathrm{~min}$. Für die präsentierten Ergebnisse waren $j=23$ bzw. $j=28$ Iterationen nötig. Die numerischen Berechnungen wurden auf einer CPU des Typs Intel Core 2 Q9550 mit $2.83 \mathrm{GHz}$ und 8 GB Arbeitsspeicher durchgeführt.

\section{Zusammenfassung}

In diesem Beitrag wurde am Beispiel induktiver Heizvorgänge ein neuartiger Ansatz zur dynamischen Optimierung vorgestellt, der sich insbesondere für die Anwendung auf Multiphysik-Probleme und Probleme auf komplexen geometrischen Gebieten eignet. Die hergeleiteten Optimalitätsbedingungen liegen dabei in Form von unbeschränkten partiellen Differentialgleichungen vor, weshalb für deren numerische Lösung auf FEM-Software zurückgegriffen wird. Als ein geeignetes Optimierungsverfahren hat sich das Gradientenverfahren herausgestellt, welches in diesem Beitrag in Kombination mit COMSOL MuLTiPHYsIcs für die numerische Lösung der dynamischen Optimierungsprobleme eingesetzt wurde.

Die Erweiterung des Ansatzes auf eine größere Klasse von Multiphysik-Problemen sowie die systematische Betrachtung von Zustandsbeschränkungen mit Hilfe geeigneter Transformationsansätze $[10,11,26]$ ist Gegenstand aktueller Arbeiten.

\section{Literatur}

1. A. Alonso Rodriguez und A. Valli. Eddy Current Approximation of Maxwell Equations: Theory, Algorithms and Applications. Springer, New York, USA, 2010.

2. H. W. Alt. Lineare Funktionalanalysis. Springer, Heidelberg, Germany, 2011.

3. B. Aschauer. Optimierung der Produktentwicklung durch Simulation: Grundlagen und Einsatzmöglichkeiten der FEM im Produktentstehungsprozess. VDM Verlag Dr. Müller, Saarbrücken, Germany, 2008.

4. A. E. Bryson und Y. C. Ho. Applied Optimal Control. Blaisdell, New York, USA, 1969.

5. P. D. Christofides. Nonlinear and Robust Control of PDE Systems. Birkhäuser, Boston, USA, 2001.

6. H. O. Fattorini. Infinite Dimensional Optimization and Control Theory. Cambridge University Press, Cambridge, UK, 1999.

7. C. Fleck und A. Schönbohm. Entwurf einer flachheitsbasierten Vorsteuerung für die induktive Erwärmung beim Thixoforming. at-Automatisierungstechnik, 9:403-410, 2009.
8. K. Graichen, M. Egretzberger und A. Kugi. Ein suboptimaler Ansatz zur schnellen modellprädiktiven Regelung nichtlinearer Systeme. at - Automatisierungstechnik, 8:447-456, 2010.

9. K. Graichen und B. Käpernick. A real-time gradient method for nonlinear model predictive control. In Frontiers of Model Predictive Control, Seiten 9-28. InTech, 2012. [Online]. Verfügbar: http://www.intechopen.com/articles/show/title/a-real-timegradient-method-for-nonlinear-model-predictive-control.

10. K. Graichen, A. Kugi, N. Petit und F. Chaplais. Handling constraints in optimal control with saturation functions and system extension. System \& Control Letters, 59(11):671-679, 2010.

11. K. Graichen und N. Petit. Incorporating a class of constraints into the dynamics of optimal control problems. Optimal Control Applications and Methods, 30(6):537-561, 2009.

12. R. F. Harrington. Time-Harmonic Electromagnetic Fields. John Wiley \& Sons, Ltd, New York, USA, 2001.

13. M. Hinze, R. Pinnau, M. Ulbrich und S. Ulbrich. Optimization with PDE Constraints. Springer, New York, USA, 2009.

14. D. E. Keyes, L. C. McInnes, C. Woodward, W. Gropp und E. Myra. Multiphysics simulations: Challenges and opportunities. International Journal of High Performance Computing Applications, 27:4-83, 2013.

15. J. L. Lions. Optimal Control of Systems Governed by Partial Differential Equations. Springer, Berlin, Germany, 1971.

16. O. Marsden, C. Bogey und C. Bailly. A study of infrasound propagation based on high-order finite difference solutions of the navier-stokes equations. Journal of the Acoustical Society of America, 135(3):1083-1095, 2014.

17. P. Monk. Finite Element Methods for Maxwell's Equations. Oxford University Press, Oxford, UK, 2003.

18. I. Neitzel. Numerical analysis of PDE-constrained optimal control problems with pointwise inequality constraints on the state and the control. PhD Thesis, Technische Universität Berlin, 2011.

19. E. Neufeld. High resolution hyperthermia treatment planning. PhD Thesis, Eidgenössische Technische Hochschule ETH Zürich, Zürich, Switzerland, 2008.

20. S. Rhein, T. Utz und K. Graichen. Optimal control of induction heating processes using FEM software. Accepted for presentation at the 14th European Control Conference (ECC), Linz, Austria, 2015.

21. V. Rudnev, D. Loveless, R. L. Cook und M. Black. Handbook of Induction Heating. Taylor \& Francis, New York, USA, 2002.

22. V. Thomée. Galerkin Finite Element Methods for Parabolic Problems. Springer, New York, USA, 2006.

23. F. Tröltzsch. Optimale Steuerung Partieller Differentialgleichungen. Vieweg+Teubner Verlag/GWV Fachverlage GmbH, Wiesbaden, Germany, 2009.

24. F. Tröltzsch und I. Yousept. PDE-constrained optimization of time-dependent 3D electromagnetic induction heating by alternating voltages. ESAIM: Mathematical Modelling and Numerical Analysis, 46:709-729, 2012.

25. T. Utz, K. Graichen und A. Kugi. Trajectory planning and receding horizon tracking control of a quasilinear diffusionconvection-reaction system. In Proceedings of the 8th IFAC Symposium on Nonlinear Control Systems (NOLCOS), Seiten 587-592, Bologna, Italy, 2010.

26. T. Utz, S. Rhein und K. Graichen. Transformation approach to constraint handling in optimal control of the heat equation. 
In Proceedings of the 19th IFAC World Congress, Seiten 91359140, Cape Town, South Africa, 2014.

27. N. Werner. Analysis and automation of the crucible-free Floating Zone (FZ) growth of silicon crystals. PhD Thesis, Leibniz Institute for Crystal Growth (IKZ), Berlin, Germany, 2014.

28. I. Yousept. Optimal control of a nonlinear coupled electromagnetic induction heating system with pointwise state constraints. Mathematics and its Applications : Annals of the Academy of Romanian Scientists, 2:45-77, 2010.

\section{Autoreninformationen}

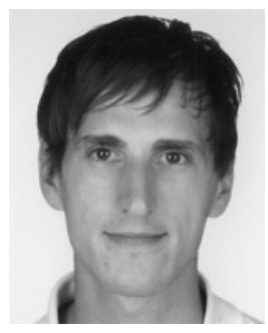

\section{M.Sc. Sönke Rhein}

Universität Ulm, Insitut für Mess-, Regelund Mikrotechnik, Albert-Einstein-Allee 41, D-89081 Ulm

soenke.rhein@uni-ulm.de

Sönke Rhein ist wissenschaftlicher Mitarbeiter am Institut für Mess-, Regel- und Mikrotechnik der Universität Ulm. Hauptarbeitsgebiet: Steuerung und Regelung verteilt-parametrischer Systeme.

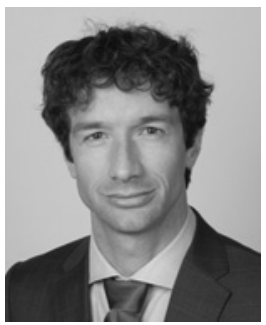

\section{Dr.techn. Tilman Utz}

Universität Ulm, Insitut für Mess-, Regelund Mikrotechnik, Albert-Einstein-Allee 41, D-89081 Ulm

tilman.utz@uni-ulm.de

Tilman Utz hat bis März 2015 als Akademischer Rat am Institut für Mess-, Regel- und Mikrotechnik der Universität Ulm gearbeitet. Hauptarbeitsgebiet: Steuerung und Regelung verteiltparametrischer Systeme.

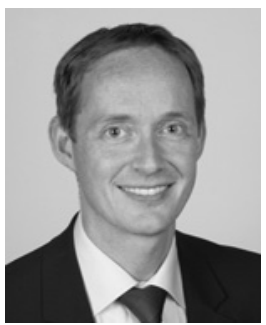

\section{Prof. Dr.-Ing. Knut Graichen} Universität Ulm, Insitut für Mess-, Regelund Mikrotechnik, Albert-Einstein-Allee 41, D-89081 Ulm knut.graichen@uni-ulm.de

Knut Graichen ist Professor am Institut für Mess-, Regel- und Mikrotechnik der Universität Ulm. Hauptarbeitsgebiete: optimale und modellprädiktive Regelung, nichtlineare Steuerungs- und Regelungsverfahren, mechatronische und verfahrenstechnische Systeme. 